\title{
Ulam stability of Volterra integral equation on a generalized metric space
}

\author{
Sorina Anamaria Ciplea and Nicolaie Lungu
}

\begin{abstract}
The aim of this paper is to give some Ulam-Hyers stability results for Volterra integral equations on a generalized metric space. In this case we consider the Volterra integral equation in the Krasnoselski-Krein and Naguno-Perron-Van Kampen conditions. Here we present only Ulam-Hyers stability for the Volterra integral equation.
\end{abstract}

Mathematics Subject Classification (2010): 45G10, 45M10, 47H10, 47J20.

Keywords: Volterra integral equations, Ulam-Hyers stability, generalized metric space, Krasnoselski-Krein conditions, Naguno-Perron-Van Kampen conditions.

\section{Introduction}

The Ulam stability is an important concept in the theory of Volterra integral equations. This problem has been studied by L.P. Castro and A. Ramos [1], N. Cădariu and V. Radu [2], S.M. Jung [3], I.A. Rus [9], [10], I.A. Rus and N. Lungu [11]. But, on a generalized metric spaces this problem has been studied in the papers [1] and [10]. In what follows we shall present Ulam-Hyers stability of a Volterra integral equation on a generalized metric space, N. Lungu [5]. Here, we consider a Volterra integral equation in the Krasnoselski-Krein and Naguno-Perron-Van Kampen conditions. In the present work we consider a generalized metric space $(X, d)$, where $d(x, y) \in \mathbb{R}_{+} \cup\{+\infty\}$ is a generalized metric on $X$. For these we need some notions and results from the generalized metric spaces theory. relation:

Let $(X, d)$ be a generalized metric space. On $X$ we have the following equivalence

$$
x \sim y \Leftrightarrow d(x, y)<\infty, \forall x, y \in X .
$$

Let $X=\bigcup_{\lambda \in \Lambda} X_{\lambda}$ be the canonical decomposition of $X$ after this equivalence relation. We denote

$$
d_{\lambda}(x, y)=\left.d(x, y)\right|_{X_{\lambda} \times X_{\lambda}}
$$


and we have that $\left(X_{\lambda}, d_{\lambda}\right)$ is a metric space $([7])$.

In this paper we need the following two theorems (see W.A.J. Luxemburg [6], I.A. Rus [7], [8]):

Theorem 1.1. Let $(X, d)$ be a generalized complete metric space and $A: X \rightarrow X$ an operator with the property:

$$
\exists \alpha \in[0,1] \text { such that } d(x, y)<\infty \Rightarrow d(A(x), A(y)) \leq \alpha d(x, y)
$$

for all $x, y \in X$.

If there exists $x_{0} \in X$ such that $d\left(x_{0}, A\left(x_{0}\right)\right)<+\infty$, then the operator $A$ has at least one fixed point.

Theorem 1.2. (Luxemburg-Jung). Let $(X, d)$ be a generalized complete metric space and his canonical decomposition $X=\bigcup X_{\lambda}$. If $A: X \rightarrow X$ ia a contraction, then the operator $A$ have in every $X_{\lambda}$, for which exists $u_{\lambda}$ such that

$$
d\left(u_{\lambda}, A\left(u_{\lambda}\right)\right)<+\infty
$$

a unique fixed point.

\section{Ulam-Hyers stability in the generalized Krasnoselski-Krein conditions}

In what follows we shall consider the following integral equation

$$
\begin{gathered}
u(x, y)=h(x, y)+\int_{0}^{x} \int_{0}^{y} f(s, t, u(s, t)) d s d t \\
f:[0, a) \times[0, b) \times \mathbb{R} \rightarrow \mathbb{R}, h:[0, a) \times[0, b) \rightarrow \mathbb{R}, \\
f \in C([0, a) \times[0, b) \times \mathbb{R}, \mathbb{R}), \\
h \in C([0, a) \times[0, b), \mathbb{R}), u \in C([0, a) \times[0, b), \mathbb{R}), \\
(x, y) \in[0, a) \times[0, b), D=[0, a) \times[0, b) .
\end{gathered}
$$

Let $X$ be the set:

$$
X=C(D)
$$

and the generalized metrics:

$$
\begin{gathered}
d: X \times X \rightarrow \mathbb{R}_{+} \cup\{+\infty\} \\
d\left(u_{1}, u_{2}\right):=\sup _{D} \frac{\left|u_{1}(x, y)-u_{2}(x, y)\right|}{(x y)^{p \sqrt{k}}}
\end{gathered}
$$

for all $u_{1}, u_{2} \in X, p>1, k>0$.

It is known that the space $(X, d)$ is a generalized complete metric space.

Let $a, b \in(0, \infty]$ and $\varepsilon>0$. In what follows we denote by $A$ the operator

$$
\begin{gathered}
A: X \rightarrow X \\
A(u)(x, y):=\text { the second part of (2.1). }
\end{gathered}
$$

Then the equation (2.1) becomes

$$
u(x, y)=A(u)(x, y)
$$


For the fixed point equation (2.4) we have:

Definition 2.1. ([10]) The equation (2.4) is Ulam-Hyers stable if there exists the positive real number $C_{f}>0$ such that, for each $\varepsilon \in \mathbb{R}_{+}^{*}$ and each solution $v$ of the inequation

$$
d(v, A v) \leq \varepsilon
$$

there exists a solution $u \in X$ of (2.4) such that

$$
d(u, v) \leq C_{f} \cdot \varepsilon .
$$

In this case we have

Theorem 2.2. We suppose that:

(i) $f: E \rightarrow \mathbb{R}$ is continuous and bounded on $E, E=D \times \mathbb{R}$;

(ii) $a<\infty, b<\infty$;

(iii) $f$ verifies the generalized Krasnoselski-Krein conditions ([4]):

$$
\begin{gathered}
\left|f\left(x, y, u_{1}\right)-f\left(x, y, u_{2}\right)\right| \leq \frac{k}{x y}\left|u_{1}-u_{2}\right|, k>0 \\
\left|f\left(x, y, u_{1}\right)-f\left(x, y, u_{2}\right)\right| \leq \frac{c}{(x y)^{\beta}}\left|u_{1}-u_{2}\right|^{\alpha}, c>0 \\
\alpha \in(0,1), \beta<\alpha, k(1-\alpha)^{2}<(1-\beta)^{2}, \beta<p \sqrt{k}, x y \neq 0, \\
p^{2} k(1-\alpha)^{2}<(1-\beta)^{2}, \text { for all }(x, y, u) \in E .
\end{gathered}
$$

Then the equation (2.4) is Ulam-Hyers stable.

Proof. We consider $X=C(D)$ and $X=\bigcup_{\lambda \in \Lambda} X_{\lambda}$. Let $v$ be a solution of the inequation (2.5) and there exists $\lambda \in \Lambda$ such that $v \in X_{\lambda}$. By Luxemburg-Jung theorem (Theorem 1.2), the equation (2.4) has a unique solution $u$ in $X_{\lambda}$.

From (2.1), (2.5), (2.6) and (2.7), we have:

$$
\begin{aligned}
|v(x, y)-u(x, y)| & \leq\left|v(x, y)-h(x, y)-\int_{0}^{x} \int_{0}^{y} f(s, t, v(s, t)) d s d t\right| \\
& +\int_{0}^{x} \int_{0}^{y}|f(s, t, v(s, t))-f(s, t, u(s, t))| d s d t .
\end{aligned}
$$

Hence, from (2.4), (2.6) and (2.7), we have

or

$$
|v(x, y)-u(x, y)| \leq|v(x, y)-A(v)(x, y)|+\int_{0}^{x} \int_{0}^{y} \frac{k}{s t}|v(s, t)-u(s, t)| d s d t
$$

$$
|v(x, y)-u(x, y)| \leq|v(x, y)-A(v)(x, y)|+\int_{0}^{x} \int_{0}^{y} k d(u, v)(s t)^{p \sqrt{k}-1} d s d t
$$

and

$$
|v(x, y)-u(x, y)| \leq|v(x, y)-A(v)(x, y)|+k d(u, v) \frac{(x y)^{p \sqrt{k}}}{p^{2} k},
$$

from where we have

$$
d(u, v) \leq \varepsilon+\frac{1}{p^{2}} d(u, v)
$$


and

then

$$
d(u, v) \leq \frac{p^{2}}{p^{2}-1} \varepsilon
$$

where

$$
d(u, v) \leq C_{f} \cdot \varepsilon
$$

$$
C_{f}=\frac{p^{2}}{p^{2}-1} .
$$

So, from Definition 2.1, the equation (2.4) is Ulam-Hyers stable.

Example 2.3. Let us consider the equation (2.1) in the Krasnoselski-Krein conditions $(2.6)+(2.7)$ and

$$
f(x, y, u)=u(x, y) x y e^{-x^{2} y^{2}}, h(x, y)=x^{2} y^{2},
$$

then $\alpha=\frac{1}{2}, \beta=\frac{1}{3}, k=1, p=2$.

In this case we have $c_{f}=\frac{p^{2}}{p^{2}-1}$ and for $p=2, c_{f}=\frac{4}{3}$, hence the equation (2.1) is Ulam-Hyers stable.

\section{Ulam-Hyers stability in the generalized Naguno-Perron-Van Kampen conditions}

In this case we consider the integral equation (2.1) in the same conditions. Let $X=C(D)$ and the generalized metrics

$$
\begin{gathered}
d: X \times X \rightarrow \mathbb{R}_{+} \cup\{+\infty\} \\
d\left(u_{1}, u_{2}\right)=\sup _{D} \frac{\left|u_{1}(x, y)-u_{2}(x, y)\right|}{(x y)^{p+1}}
\end{gathered}
$$

for all $u_{1}, u_{2} \in X, p>-1$.

It is known that the space $(X, d)$ is a generalized complete metric space. Here, we consider the stability of the equation (2.4) in the generalized Naguno-Perron-Van Kampen conditions.

Theorem 3.1. If we have

(i) $f: E \rightarrow \mathbb{R}$ is continuous and bounded on $E$;

(ii) $a<+\infty, b<+\infty$;

(iii) $f$ verifies the generalized Naguno-Perron-Van Kampen conditions ([12]):

$$
\begin{gathered}
|f(x, y, u)| \leq \alpha(x y)^{p}, p>-1, \alpha>0 . \\
\left|f\left(x, y, u_{1}\right)-f\left(x, y, u_{2}\right)\right| \leq \frac{c}{(x y)^{r}}\left|u_{1}-u_{2}\right|^{q}, q \geq 1, c>0, \\
p q+q-r=p, x y \neq 0, \rho=\frac{c(2 \alpha)^{q-1}}{(p+1)^{2 q}}<1, \text { for all }(x, y, u) \in E .
\end{gathered}
$$

Then the equation (2.4) is Ulam-Hyers stable. 
Proof. Evidently, in the conditions Naguno-Perron-Van Kampen, by Luxemburg-Jung theorem (Theorem 1.2), the equation (2.4) has a unique solution $u$ in $X_{\lambda}$.

First we observe that

$$
|v(x, y)-u(x, y)| \leq \frac{2 \alpha}{(p+1)^{2}}(x y)^{p+1} .
$$

From (2.1), (2.5), (3.2), (3.3) we have

$$
\begin{aligned}
|v(x, y)-u(x, y)| & \leq\left|v(x, y)-h(x, y)-\int_{0}^{x} \int_{0}^{y} f(s, t, v(s, t)) d s d t\right| \\
& +\int_{0}^{x} \int_{0}^{y}|f(s, t, v(s, t))-f(s, t, u(s, t))| d s d t .
\end{aligned}
$$

From (3.3) we have

$$
\begin{gathered}
|v(x, y)-u(x, y)| \leq|v(x, y)-A(v)(x, y)|+\int_{0}^{x} \int_{0}^{y} \frac{c}{(s t)^{r}}|v(s, t)-u(s, t)|^{q} d s d t \\
\leq|v(x, y)-A(v)(x, y)|+\int_{0}^{x} \int_{0}^{y} \frac{c}{(s t)^{r}} \cdot \frac{|v(s, t)-u(s, t)|}{(s t)^{p+1}} \cdot \frac{|v(s, t)-u(s, t)|^{q-1}}{(s t)^{-p-1}} d s d t \\
\leq|v(x, y)-A(v)(x, y)|+c d(u, v) \int_{0}^{x} \int_{0}^{y} \frac{(2 \alpha)^{q-1}}{(p+1)^{2(q-1)}}(s t)^{p q+q-r} d s d t .
\end{gathered}
$$

Then we have

$$
d(u, v) \leq d(v, A(v))+\rho d(u, v)
$$

and

$$
d(u, v) \leq \frac{\varepsilon}{1-\rho}
$$

then

$$
d(u, v) \leq C_{f} \cdot \varepsilon
$$

where

$$
C_{f}=\frac{1}{1-\rho} .
$$

From Definition 2.1, the equation (2.4) is Ulam-Hyers stable.

Remark 3.2. For every $\lambda \in \Lambda$ there exists at least a solution $v$ of (2.5) in $X_{\lambda}$ and for each $v$ exists a unique solution $u$ of (2.4) which is Ulam-Hyers stable.

Remark 3.3. It is possible that the inequation (2.5) do not have a solution, but in this case the equation (2.4) is Ulam-Hyers stable.

Example 3.4. Let us consider the equation (2.1) in the Naguno-Perron-Van Kampen conditions $(3.2)+(3.3), p>-1, r=1, q \geq 1$.

In this case $c_{f}=\frac{1}{1-\rho}$, where $\rho=\frac{c(2 \alpha)^{q-1}}{(p+1)^{2 q}}$ and the equation (2.1) is UlamHyers stable. If $\rho=1$ then the equation (2.1) is Ulam-Hyers instable. 


\section{References}

[1] Castro, L.P., Ramos, A., Hyers-Ulam-Rassias stability for a class of nonlinear Volterra integral equations, Banach J. Math. Anal., 3(2009), no. 1, 36-43.

[2] Cădariu, L., Radu, V., The fixed points method for the stability of some functional equations, Carpathian J. Math., 23(2007), no. 1-2, 63-72.

[3] Jung, S.M., A fixed point approach to the stability of a Volterra integral equation, Fixed Point Theory and Applications, 2007, Art. ID57064, 9 pages.

[4] Krasnoselski, M.A., Krein, S.G., Ob odnom classe theorem edinstvenosti dlia uravnenia $y^{\prime}=f(x, y)$, Uspehi Math. Nauk, 1(67), 11(1956), 209-213.

[5] Lungu, N., Hyperbolic-type inequalities on generalized metric spaces, Carpathian J. Math., 24(2008), no. 3, 341-347.

[6] Luxemburg, W.A.J., On the convergence of successive approximations in the theory of ordinary differential equations II, Indag. Math., 20(1958), 540-546.

[7] Rus, A.I., Principii şi aplicaţii ale teoriei punctului fix, Ed. Dacia, Cluj-Napoca, 1979.

[8] Rus, A.I., Ulam stability of ordinary differential equations, Stud. Univ. Babeş-Bolyai Math., 54(2009), no. 4, 125-133.

[9] Rus, A.I., Gronwall lemma approach to the Hyers-Ulam-Rassias stability of an integral equation, in: Nonlinear Analysis and Variational Problems, Springer, 2009, 147-152.

[10] Rus, A.I., Remarks on Ulam stability of the operatorial equations, Fixed Point Theory, 10(2009), no. 2, 305-320.

[11] Rus, A.I., Lungu, N., Ulam stability of a nonlinear hyperbolic partial differential equation, Carpathian J. Math., 24(2008), no. 3, 403-408.

[12] Wong, I.S.W., On the convergence of successive approximations in the Darboux problem, Ann. Polon. Math., 18(1966), 329-336.

Sorina Anamaria Ciplea

Technical University of Cluj-Napoca

Department of Management and Technology

28 Memorandumului Street, 400114

Cluj-Napoca, Romania

e-mail: sorina.ciplea@ccm.utcluj.ro

Nicolaie Lungu

Technical University of Cluj-Napoca

Department of Mathematics

28 Memorandumului Street

400114 Cluj-Napoca, Romania

e-mail: nlungu@math .utcluj.ro 